\title{
On surface braids of index four with at most two crossings
}

\author{
by \\ Teruo Nagase and Akiko Shima (Kanagawa) \\ Dedicated to Professor Yukio Matsumoto for his sixtieth birthday
}

\begin{abstract}
Let $\Gamma$ be a 4 -chart with at most two crossings. We show that if the closure of the surface braid obtained from $\Gamma$ is one 2 -sphere, then the sphere is a ribbon surface.
\end{abstract}

1. Introduction. Kamada showed that any 3-chart can be modified by C-moves to a 3-chart without white vertices (see [3]). Nagase and Hirota showed that any 4-chart with at most one crossing can be modified by C-moves to a chart without white vertices (see [4]). Kamada showed that for any ribbon surface, for some positive integer $n$ there exists an $n$-chart without white vertices such that the ribbon surface is ambient isotopic to the closure of the surface braid obtained from it (see [3, Proposition 20]). Conversely, if a chart can be modified by C-moves to a chart without white vertices, then the closure of the surface braid obtained from it is ambient isotopic to a ribbon surface. Hence the closure of the surface braid obtained from a 4-chart with at most one crossing is ambient isotopic to a ribbon surface.

Let $\Gamma$ be a 4-chart with exactly two crossings. Aiba and Nagase showed that if $\Gamma$ is a minimal 4-chart with exactly one special house, then each connected component of $\Gamma$ is a free edge, a hoop, a 4-chart as shown in Figure 1, or its reflection. Here, a house means a complementary domain of the subgraph consisting of even labeled edges and their vertices, a special house means a house containing crossings, a free edge $E$ means an edge such that each vertex of $E$ has degree one, and a hoop means an edge without vertices.

2000 Mathematics Subject Classification: Primary 57Q45; Secondary 57Q35.

The second author is partially supported by Grant-in-Aid for Scientific Research (No. 16540082), Ministry of Education, Science and Culture, Japan. 
The 4-chart in Figure 1 has eight black vertices, where a black vertex means a vertex of degree one. On the other hand, if the closure of the surface braid obtained from a 4-chart represents a 2 -sphere, then the chart has exactly six black vertices. Therefore if $\Gamma$ is a connected minimal 4-chart with exactly two crossings and one special house, then the closure of the surface braid obtained from $\Gamma$ is either a ribbon surface, a connected closed surface of genus $g \geq 1$, or a disconnected closed surface.

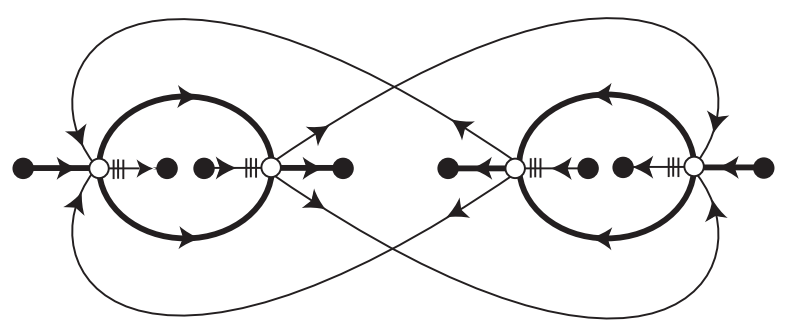

Fig. 1. The thick lines are edges of label 2. The other lines are edges of label 1 or 3.

The following is the main result of this paper.

THEOREM 1.1. Let $\Gamma$ be a 4-chart with at most two crossings. If the closure of the surface braid obtained from $\Gamma$ is one 2 -sphere, then $\Gamma$ can be modified by $C$-moves to a 4-chart without white vertices. Hence the sphere is a ribbon surface.

2. Definitions and preliminaries. In this section, we investigate distinguished arcs and terminal edges of label 2 .

Let $n$ be a positive integer. An $n$-chart is an oriented labeled graph in a disk, which may be empty or have closed edges without vertices called hoops, satisfying the following four conditions:

(i) Every vertex has degree 1, 4, or 6.

(ii) The labels of edges are in $\{1,2, \ldots, n-1\}$.

(iii) In a small neighborhood of each vertex of degree 6 , there are six short arcs, three consecutive arcs are oriented inward and the other three outward, and those six arcs are labeled $i$ and $i+1$ alternately for some $i$, where the orientation and label of each arc are inherited from the edge containing the arc.

(iv) For each vertex of degree 4, diagonal edges have the same label and are oriented coherently, and the labels $i$ and $j$ of the diagonals satisfy $|i-j|>1$.

A vertex of degree 1, 4, and 6 is called a black vertex, a crossing, and a white vertex respectively. To make the argument simple, we assume that the charts lie on the 2-sphere instead of the disk. 
Among the six short arcs in a small neighborhood of a white vertex, the center arc of any three consecutive arcs oriented inward or outward is called a middle arc of the white vertex. There are two middle arcs in a small neighborhood of each white vertex. A middle arc of odd label is called a distinguished arc. We mark it as in Figure 2. If an edge contains a distinguished arc containing a white vertex $w$, then the edge is called a distinguished edge at $w$. An edge is called free if it has two black vertices; terminal if it contains one black vertex and one white vertex; and a loop if it contains only one vertex.
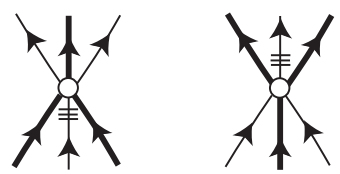

Fig. 2. The thick lines are edges of label 2. The other lines are edges of label 1 or 3.

REMARK 2.1. Around each white vertex, there exists only one distinguished arc.

Let $\Gamma$ be a connected 4-chart, and $\Gamma_{2}$ the subgraph of $\Gamma$ consisting of edges of label 2 and their vertices. Then a complementary domain of $\Gamma$ and $\Gamma_{2}$ are called a room and a house, respectively. Note that all rooms are open disks. In general, a house and a room of each component of a 4-chart are called a house and a room of the 4-chart, respectively.

Lemma $2.2([1])$. Let $\Gamma$ be a connected 4-chart, $t(\Gamma)$ the number of terminal edges of label 2 , and $w(\Gamma)$ the number of white vertices. For each natural number $i$, let $n_{i}$ be the number of houses with exactly $i$ boundary components each. Then

$$
w(\Gamma)-t(\Gamma)=2\left(n_{1}-2\right)-2 \sum_{i \geq 3} n_{i}(i-2) .
$$

A $C$-move is a local modification of charts in a disk as shown in Lemma 16 of [3] (cf. Figures 19 and 20 in Chapter 3 of [2]). We show some C-moves in Figure 3. Two charts are $C$-move equivalent if there exists a finite sequence of C-moves which modify one of the charts to the other.

For each chart $\Gamma$, let $c(\Gamma), w(\Gamma)$ and $f(\Gamma)$ be the number of crossings, of white vertices, and of free edges respectively. The triplet $(c(\Gamma), w(\Gamma)$, $-f(\Gamma))$ is called the complexity of the chart. A chart is called minimal if its complexity is lexicographically minimal among the charts C-move equivalent to it.

REMARK 2.3. Let $\Gamma$ be a minimal 4-chart. Then

(i) no edge contains two distinguished arcs, 
(ii) any terminal edge contains a middle arc, in particular, an odd labeled terminal edge is a distinguished edge (see the C-III-1 move in Figure 3).
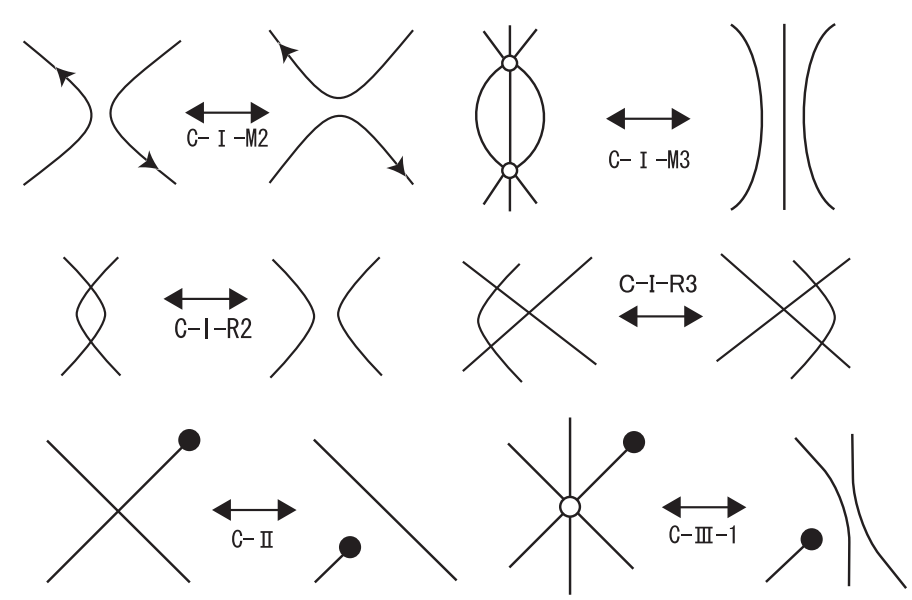

Fig. 3. In the left figure of a C-III-1 move, the edge containing the black vertex does not contain a middle arc.

It is easy to prove the following lemma.

LEMMA 2.4. Each component of a minimal 4-chart is a minimal 4-chart.

TERMinOLOGY. If a vertex or an edge is contained in the closure of a room or a house, then we say that the vertex or the edge belongs to the room or the house, or that the room or the house possesses the vertex or the edge.

Let $\Gamma$ be a connected 4-chart, $R$ a room of $\Gamma$, and $X_{R}$ the closure of $R$. Let $D$ be a disk, and $\bar{P}_{1}, \ldots, \bar{P}_{n}$ points on the boundary of $D, \partial D$, situated in this order. The points split $\partial D$ into $n$ arcs $\bar{A}_{1}, \ldots, \bar{A}_{n}$ where $\bar{P}_{i}$ and $\bar{P}_{i+1}$ are the end points of $\bar{A}_{i}$, with $\bar{P}_{n+1}=\bar{P}_{1}$. Let $g: D \rightarrow X_{R}$ be a continuous surjective map which:

(i) maps the interior of $D$ homeomorphically onto $R$, and hence $\partial D$ onto $\partial R$,

(ii) maps the interior of each arc $\bar{A}_{i}$ homeomorphically onto the interior of an edge belonging to the room $R$.

Then the set $\left\{g: D \rightarrow X_{R} ; \bar{P}_{1}, \ldots, \bar{P}_{n} ; \bar{A}_{1}, \ldots, \bar{A}_{n}\right\}$ is called an associated set of the room $R$. The set $\left\{g: D \rightarrow X_{R} ; \bar{A}_{1}, \ldots, \bar{A}_{n}\right\}$ is also called an associated set of $R$. Similarly, we can define an associated set of a house with connected boundary. 
Let $\Gamma$ be a chart, and $X_{R}$ the closure of a room $R$ of $\Gamma$. Let $A_{1}$ and $A_{2}$ be different edges in $X_{R}$. The pair $\left(A_{1}, A_{2}\right)$ is said to be admissible with respect to a disk $E$ in $X_{R}$ if:

(i) $E \cap \partial R$ consists of two disjoint $\operatorname{arcs} \alpha_{1}, \alpha_{2}$ with $\alpha_{i} \subset A_{i}$ for $i=1,2$.

(ii) For any orientation of $E$, there exists some $i \in\{1,2\}$ such that the orientation induced from the disk does not coincide with the orientation induced from $A_{i}$.

Let $A, A^{\prime}$ and $A^{\prime \prime}$ be edges which belong to a room $R$ such that $A$ is a terminal edge of label 2 , and the labels of $A^{\prime}$ and $A^{\prime \prime}$ are odd (the case of $A^{\prime}=A^{\prime \prime}$ is not excluded). Let $\left\{g: D \rightarrow X_{R} ; \bar{P}_{1}, \ldots, \bar{P}_{n} ; \bar{A}_{1}, \ldots, \bar{A}_{n}\right\}$ be an associated set of $R$ with $A=g\left(\bar{A}_{1}\right)$. We can assume that $A^{\prime}=g\left(\bar{A}_{i}\right)$, $A^{\prime \prime}=g\left(\bar{A}_{j}\right)$, and $i<j$. The triplet $\left(A^{\prime}, A, A^{\prime \prime}\right)$ is said to be semi-reducible with respect to a disk $E$ if it satisfies condition (1) below, and reducible with respect to $E$ if it satisfies (1) and (2).

(1) $A$ splits $E$ into two disks, say $E_{1}$ and $E_{2}$, so that the pair $\left(A, A^{\prime}\right)$ is admissible with respect to one of them, and $\left(A, A^{\prime \prime}\right)$ is admissible with respect to the other.

(2) If $g\left(\bar{A}_{k} \cap \bar{A}_{k+1}\right)$ is a crossing for some $k$ with $i \leq k<j$, then the triplet $\left(g\left(\bar{A}_{k}\right), A, g\left(\bar{A}_{k+1}\right)\right)$ is not semi-reducible.

Lemma 2.5 ([4, Lemma 1$])$. For any minimal 4-chart, there is no reducible triplet.

A special pair is an admissible pair with a common crossing. A special house is a house containing a crossing.

Lemma 2.6. Let $\Gamma$ be a connected minimal 4-chart. If a room $R$ possesses exactly $m$ special pairs, then it possesses at most $m$ terminal edges of label 2.

Proof. Let $\left\{g: D \rightarrow X_{R} ; \bar{A}_{1}, \ldots, \bar{A}_{n}\right\}$ be an associated set of $R$. We prove the lemma by contradiction. Suppose that the room possesses more than $m+1$ terminal edges of label 2 . Then there exist two terminal edges $g\left(\bar{A}_{i}\right)$ and $g\left(\bar{A}_{j}\right)$ of label 2 with $i<j$ such that $g\left(\bar{A}_{i+1}\right)$ and $g\left(\bar{A}_{j-1}\right)$ are of odd label, and $\left(g\left(\bar{A}_{k}\right), g\left(\bar{A}_{k+1}\right)\right)$ is a special pair for any $k=i+1$, $i+2, \ldots, j-2$. Now $\left(g\left(\bar{A}_{i+1}\right), g\left(\bar{A}_{j-1}\right)\right)$ is admissible. For, if not, we can increase the number of free edges by a C-I-M2 move between the terminal edges $g\left(\bar{A}_{i}\right)$ and $g\left(\bar{A}_{j}\right)$. Therefore the triplet $\left(g\left(\bar{A}_{i+1}\right), g\left(\bar{A}_{i}\right), g\left(\bar{A}_{j-1}\right)\right)$ is reducible. This contradicts Lemma 2.5 .

The following lemma is a generalization of Proposition 1 in [4].

Lemma 2.7. Let $\Gamma$ be a connected minimal 4-chart. If a house of $\Gamma$ contains exactly $n$ crossings, then the house possesses at most $2 n$ terminal edges of label 2. In particular, no non-special house possesses a terminal edge of label 2. 
Proof. If a house is non-special, then it possesses no terminal edge of label 2 by Proposition 1 in [4]. If a house is special, then it contains crossings. For each crossing, there are two special pairs, so if the house contains exactly $n$ crossings, then it contains exactly $2 n$ special pairs. By Lemma 2.6, the house possesses at most $2 n$ terminal edges of label 2 .

Let $\Gamma$ be a connected 4-chart, and $H$ a house of $\Gamma$. Let $m_{1}$ be the number of distinguished arcs in the closure of $H$, and $m_{2}$ the number of terminal edges of label 2 in that closure. Then $m_{1}-m_{2}$ is denoted by $d(H)$. By Remark 2.1, the number of white vertices of $\Gamma$ is equal to the number of distinguished arcs of $\Gamma$. Hence the sum of $d(H)$ for all houses is equal to $w(\Gamma)-t(\Gamma)$ :

$$
w(\Gamma)-t(\Gamma)=\sum_{H: \text { house }} d(H) .
$$

Lemma 2.8. Let $\Gamma$ be a connected minimal 4-chart, and $H$ a non-special house of $\Gamma$. If the boundary of $H$ is connected, then $H$ possesses an even number of distinguished arcs, at least two. Moreover, $d(H) \geq 2$.

Proof. Let $H$ be a non-special house with connected boundary. By Lemma 2.7, $H$ possesses no terminal edge of label 2. Lemma 4 in [4] shows that $H$ possesses an even number of distinguished arcs. By Proposition 4 in [4], $H$ possesses at least two distinguished arcs. Since $H$ possesses no terminal edge of label 2 , it follows that $d(H) \geq 2$.

In [4], Nagase and Hirota investigate edges near a terminal edge of label 2.

Lemma 2.9 ([4, Lemma 3]). Let $\Gamma$ be a minimal 4-chart, and $B, B^{\prime}$ edges containing the same white vertex $w$ such that $B$ is terminal of label 2 , and the label of $B^{\prime}$ is odd. If $B^{\prime}$ is not a distinguished edge at $w$, then it contains a crossing or is a distinguished edge at the other white vertex of itself.

Lemma 2.10 ([4, Lemma 6$])$. In a minimal 4-chart, there exists no room whose boundary consists of exactly two edges with different parities and with the odd labeled edge containing no distinguished arc.

Lemma 2.11. Let $\Gamma$ be a connected minimal 4-chart, and $H$ a special house of $\Gamma$. If the boundary of $H$ is connected, and if $H$ contains exactly one crossing, then $d(H) \geq 0$. Moreover, if $H$ possesses distinguished arcs, then $d(H) \geq 1$.

Proof. Let $B_{1}, B_{2}, B_{3}, B_{4}$ be the odd labeled edges containing the crossing which belong to $H$. They separate $H$ into four components, say $H_{1}, H_{2}$, $H_{3}, H_{4}$. Each $H_{i}$ possesses at most one special pair. By Lemma 2.7, each $H_{i}$ possesses at most one terminal edge of label 2. Suppose that $H_{i}$ possesses one terminal edge of label 2 , say $A$. Let $C_{1}, C_{2}$ be the odd labeled edges which belong to $H_{i}$ and contain the white vertex of $A$. Since $H$ contains only one 
crossing, $C_{1}$ or $C_{2}$ does not contain a crossing. If neither of them does, then both contain distinguished arcs by Lemma 2.9. This is impossible. Suppose that only one of $C_{1}$ and $C_{2}$, say $C_{1}$, contains a crossing. Let $R$ be the component of $H \backslash C_{2}$ with no crossing. Let $\left\{g: D \rightarrow X_{R} ; \bar{P}_{1}, \ldots, \bar{P}_{n} ; \bar{A}_{1}, \ldots, \bar{A}_{n}\right\}$ be an associated set of $R$ such that $g\left(\bar{A}_{1}\right)=C_{2}$ and $C_{2}$ is the distinguished edge at $g\left(\bar{P}_{1}\right)$. Then $R$ possesses two distinguished arcs. If not, then $\left(g\left(\bar{A}_{k}\right), g\left(\bar{A}_{k+1}\right)\right)$ is not admissible for $2 \leq k \leq n-1$, and $\left(g\left(\bar{A}_{2}\right), g\left(\bar{A}_{n}\right)\right)$ is not admissible. However, since $\left(C_{2}, g\left(\bar{A}_{2}\right)\right)$ is not admissible, and $\left(C_{2}, g\left(\bar{A}_{n}\right)\right)$ is, it follows that $\left(g\left(\bar{A}_{2}\right), g\left(\bar{A}_{n}\right)\right)$ is admissible. This is a contradiction. Therefore $R$ possesses at least two distinguished arcs, and if $H_{i}$ possesses one terminal edge of label 2, then it contains at least two distinguished arcs. Hence $d(H) \geq 0$. Moreover, if $H$ possesses distinguished arcs, then $d(H) \geq 1$.

3. Saturated 4-charts. In this section, we show that if $\Gamma$ is a minimal 4-chart with at most two crossings, then $\Gamma$ is a "saturated" 4-chart.

We say that a connected 4-chart $\Gamma$ with white vertices is saturated if it satisfies the following two conditions:

(i) A house of $\Gamma$ possesses distinguished arcs if and only if it is nonspecial with connected boundary.

(ii) If a house possesses distinguished arcs, then it possesses exactly two such arcs.

In general, we say that a 4-chart is saturated if each of its components is a free edge, a hoop, or a saturated 4-chart.

Nagase and Hirota showed that a minimal 4-chart with at most one crossing has no white vertices (see [4, Main Theorem]). Such a chart can be modified to a chart consisting of free edges and hoops by using C-II, C-I-R2 and C-I-R3 moves. This implies the following lemma.

Lemma 3.1. Any minimal 4-chart with at most one crossing is saturated.

Lemma 3.2 ([1, Main Theorem $])$. Let $\Gamma$ be a minimal 4-chart with exactly two crossings. If $\Gamma$ has only one special house, then each component of $\Gamma$ is a free edge, a hoop, the 4-chart of Figure 1 or its reflection.

Let $\Gamma$ be the 4 -chart of Figure 1 or its reflection. Then $\Gamma$ is saturated. By Lemma 3.2, we have the following lemma.

Lemma 3.3. Let $\Gamma$ be a minimal 4-chart with exactly two crossings. If $\Gamma$ has only one special house, then $\Gamma$ is saturated.

We wish to show that any minimal 4-chart with at most two crossings is saturated. To do this, it suffices to consider a connected minimal 4-chart with two special houses. 
LEMma 3.4. Let $\Gamma$ be a connected minimal 4-chart with exactly two crossings and with two special houses. Then

$$
w(\Gamma)-t(\Gamma) \geq 2\left(n_{1}-2\right)
$$

where $n_{1}$ is the number of houses with connected boundary, $w(\Gamma)$ is the number of white vertices, and $t(\Gamma)$ is the number of terminal edges of label 2. Moreover:

(i) If there exists a non-special house $H_{0}$ with connected boundary and with $d\left(H_{0}\right) \geq 4$, then $w(\Gamma)-t(\Gamma) \geq 2\left(n_{1}-2\right)+2$.

(ii) If there exists a non-special house $H_{0}$ with disconnected boundary and with $d\left(H_{0}\right) \geq 1$, then $w(\Gamma)-t(\Gamma) \geq 2\left(n_{1}-2\right)+1$.

To prove Lemma 3.4, we need the following claim.

Claim. Let $\Gamma$ be a 4-chart as in Lemma 3.4. Let $n$ be the number of non-special houses with connected boundary. Then $D(\Gamma) \geq 2 n$. Moreover,

$$
D(\Gamma) \geq \begin{cases}2 n+2 & \text { if } \Gamma \text { is as in Lemma 3.4(i), } \\ 2 n+1 & \text { if } \Gamma \text { is as in Lemma 3.4(ii). }\end{cases}
$$

Here, $D(\Gamma)$ is the sum of $d(H)$ for all non-special houses $H$.

Proof of Claim. Let $H_{1}^{\prime}, \ldots, H_{n}^{\prime}$ be all non-special houses with connected boundary. By Lemma 2.8, $d\left(H_{i}^{\prime}\right) \geq 2$ for all $i$. By Lemma 2.7, no non-special house $H$ possesses a terminal edge of label 2. Hence $d(H) \geq 0$. Therefore

$$
D(\Gamma) \geq \sum_{i=1}^{n} d\left(H_{i}^{\prime}\right) \geq 2 n .
$$

Suppose that $\Gamma$ is as in Lemma 3.4(i). Then $d\left(H_{j}^{\prime}\right) \geq 4$ for some $j$. Hence

$$
D(\Gamma) \geq d\left(H_{j}^{\prime}\right)+\sum_{1 \leq i \leq n, i \neq j} d\left(H_{i}^{\prime}\right) \geq 4+2(n-1)=2 n+2 .
$$

Suppose now that $\Gamma$ is as in Lemma 3.4(ii). Then there exists a nonspecial house $H_{0}$ such that $\partial H_{0}$ is disconnected and $d\left(H_{0}\right) \geq 1$. Hence $D(\Gamma) \geq d\left(H_{0}\right)+\sum_{i=1}^{n} d\left(H_{i}^{\prime}\right) \geq 1+2 n$. This completes the proof of Claim.

Let $\Gamma$ be a 4 -chart as in Lemma 3.4. Set

$$
\varepsilon= \begin{cases}2 & \text { if } \Gamma \text { is as in Lemma 3.4(i), } \\ 1 & \text { if } \Gamma \text { is as in Lemma 3.4(ii), } \\ 0 & \text { otherwise. }\end{cases}
$$

By the above claim, we have $D(\Gamma) \geq 2 n+\varepsilon$.

Proof of Lemma 3.4. Let $H_{1}, H_{2}$ be the two special houses of $\Gamma$. Since $\Gamma$ has exactly two crossings, each $H_{i}$ contains only one crossing. By the above 
claim,

$$
w(\Gamma)-t(\Gamma)=d\left(H_{1}\right)+d\left(H_{2}\right)+D(\Gamma) \geq d\left(H_{1}\right)+d\left(H_{2}\right)+2 n+\varepsilon .
$$

There are three possibilities:

(a) The boundaries of $H_{1}$ and $H_{2}$ are connected.

(b) One of $\partial H_{1}$ and $\partial H_{2}$ is connected, the other is disconnected.

(c) The boundaries of $H_{1}$ and $H_{2}$ are disconnected.

In case (a), by Lemma $2.11, d\left(H_{i}\right) \geq 0$ for $i=1,2$. Since the number of special houses with connected boundary is two, we have $n_{1}=n+2$. Hence $w(\Gamma)-t(\Gamma) \geq 2\left(n_{1}-2\right)+\varepsilon$.

In case (b), we may assume that $\partial H_{1}$ is connected and $\partial H_{2}$ is not. By Lemma 2.11, $d\left(H_{1}\right) \geq 0$. Since $H_{2}$ contains only one crossing, it possesses at most two terminal edges by Lemma 2.7. Hence $d\left(H_{2}\right) \geq-2$. Since the number of special houses with connected boundary is 1 , we have $n_{1}=n+1$. Therefore

$$
w(\Gamma)-t(\Gamma) \geq 0-2+2\left(n_{1}-1\right)+\varepsilon=2\left(n_{1}-2\right)+\varepsilon .
$$

In case (c), we have $d\left(H_{i}\right) \geq-2$ for $i=1,2$ in a similar way to the case above. Since there is no special house with connected boundary, we have $n_{1}=n$. Therefore

$$
w(\Gamma)-t(\Gamma) \geq-2-2+2 n_{1}+\varepsilon=2\left(n_{1}-2\right)+\varepsilon .
$$

This completes the proof of Lemma 3.4.

LEMMA 3.5. Let $\Gamma$ be a connected minimal 4-chart with exactly two crossings and with two special houses. Then the boundary of each house is connected or has two components.

Proof. For each natural number $i$, let $n_{i}$ be the number of houses with exactly $i$ boundary components each. Suppose that there exists a house $H$ such that $\partial H$ has more than two components. Then $n_{i} \geq 1$ for some $i \geq 3$. By Lemma 2.2,

$$
w(\Gamma)-t(\Gamma) \leq 2\left(n_{1}-2\right)-2 n_{i}(i-2) \leq 2\left(n_{1}-2\right)-2=2\left(n_{1}-3\right) .
$$

This contradicts Lemma 3.4.

LEMmA 3.6. Let $\Gamma$ be a connected minimal 4-chart with exactly two crossing and with two special houses. If there exists a special house which possesses distinguished arcs, then

$$
w(\Gamma)-t(\Gamma) \geq 2\left(n_{1}-2\right)+1
$$

where $n_{1}$ is the number of houses with connected boundary.

Proof. Let $n$ be the number of non-special houses with connected boundary. Let $H_{1}, H_{2}$ be the special houses of $\Gamma$. Suppose that $H_{1}$ possesses distinguished arcs. By the Claim, $D(\Gamma) \geq 2 n$. 
Case 1: $\partial H_{1}$ is connected. Since $H_{1}$ possesses at least one distinguished arc, $d\left(H_{1}\right) \geq 1$ by Lemma 2.11 . Suppose that $\partial H_{2}$ is connected. Then there are exactly two special houses with connected boundary, and $n_{1}=n+2$. By Lemma 2.11, $d\left(H_{2}\right) \geq 0$ and we have

$$
w(\Gamma)-t(\Gamma)=d\left(H_{1}\right)+d\left(H_{2}\right)+D(\Gamma) \geq 1+0+2 n=2\left(n_{1}-2\right)+1 .
$$

Suppose now that $\partial H_{2}$ is disconnected. Then $n_{1}=n+1$. Since $H_{2}$ contains exactly one crossing, by Lemma 2.7 it possesses at most two terminal edges of label 2 . Hence $d\left(H_{2}\right) \geq-2$ and we have

$$
w(\Gamma)-t(\Gamma)=d\left(H_{1}\right)+d\left(H_{2}\right)+D(\Gamma) \geq 1-2+2 n=2\left(n_{1}-2\right)+1 .
$$

CASE 2: $\partial H_{1}$ is disconnected. Since $H_{1}$ possesses a distinguished arc, we have $d\left(H_{1}\right) \geq-1$ in a similar way to the case above. Suppose that $\partial H_{2}$ is connected. Then $n_{1}=n+1$. By Lemma 2.11, $d\left(H_{2}\right) \geq 0$ and we have

$$
w(\Gamma)-t(\Gamma)=d\left(H_{1}\right)+d\left(H_{2}\right)+D(\Gamma) \geq-1+0+2 n=2\left(n_{1}-2\right)+1 .
$$

Suppose now that $\partial H_{2}$ is disconnected. Then $n_{1}=n$. Similarly to the above, $d\left(H_{2}\right) \geq-2$ and we have

$$
w(\Gamma)-t(\Gamma)=d\left(H_{1}\right)+d\left(H_{2}\right)+D(\Gamma) \geq-1-2+2 n=2\left(n_{1}-2\right)+1 .
$$

Theorem 3.7. Any minimal 4-chart with at most two crossings is saturated.

Proof. Let $\Gamma$ be a minimal 4-chart with at most two crossings. If each component of $\Gamma$ has at most one crossing, then $\Gamma$ is saturated by Lemmas 2.4 and 3.1. Hence we may assume that $\Gamma$ is connected and has exactly two crossings. By Lemma 3.3, we may also assume that $\Gamma$ has two special houses. By Lemma 3.5, the boundary of each house is connected or has two components. Lemma 2.2 yields

$$
w(\Gamma)-t(\Gamma)=2\left(n_{1}-2\right)
$$

where $n_{1}$ is the number of houses with connected boundary. By Lemma 2.8, if a non-special house with connected boundary possesses distinguished arcs, then it possesses at least $2 k$ such $\operatorname{arcs}(k \geq 1)$. Suppose that $\Gamma$ is not saturated. Then there exists a house $H_{1}$ satisfying one of the following conditions:

(i) $H_{1}$ is non-special with connected boundary, and possesses at least four distinguished arcs.

(ii) $H_{1}$ is non-special with two boundary components, and possesses distinguished arcs.

(iii) $H_{1}$ is special and possesses distinguished arcs.

In case (i), Lemma 2.7 shows that $H_{1}$ possesses no terminal edge of label 2. Hence $d\left(H_{1}\right) \geq 4$. By Lemma 3.4(i), we then have $w(\Gamma)-t(\Gamma) \geq$ $2\left(n_{1}-2\right)+2$. This is a contradiction. 
In case (ii), Lemma 2.7 shows that $H_{1}$ possesses no terminal edge of label 2. Hence $d\left(H_{1}\right) \geq 1$, and then $w(\Gamma)-t(\Gamma) \geq 2\left(n_{1}-2\right)+1$ by Lemma 3.4(ii). This is a contradiction.

In case (iii), $w(\Gamma)-t(\Gamma) \geq 2\left(n_{1}-2\right)+1$ by Lemma 3.6. This is a contradiction.

Lemma 3.8. Let $\Gamma$ be a saturated connected minimal 4-chart. If a special house $H$ of $\Gamma$ contains exactly one crossing, then $H$ possesses no terminal edges of label 2. Moreover, $d(H)=0$.

Proof. Suppose that a terminal edge $B$ of label 2 belongs to $H$. Let $w$ be the white vertex of $B$. Since $\Gamma$ is saturated, $H$ possesses no distinguished arcs. Hence no loop with vertex $w$ has odd label. So, there exist two odd labeled edges $B_{1}, B_{2}$ such that $B_{i}$ belongs to $H$ and $w \in B_{i}$ for $i=1$, 2. Since $H$ possesses no distinguished $\operatorname{arcs}, B_{i}$ is a distinguished edge at neither of its vertices for $i=1,2$. By Lemma 2.9, $B_{1}$ and $B_{2}$ contain crossings. However, these crossings are not different. This contradicts the fact that $H$ contains exactly one crossing. Therefore $H$ possesses no terminal edges of label 2 and $d(H)=0$.

Lemma 3.9. Let $\Gamma$ be a connected minimal 4-chart with exactly two crossings. If a special house $H_{1}$ of $\Gamma$ contains exactly one crossing, then the boundary of $H_{1}$ is connected.

Proof. By Theorem 3.7, $\Gamma$ is saturated. Let $H_{1}$ be a special house containing exactly one crossing. Suppose that $\partial H_{1}$ is disconnected. By Lemmas 2.2 and 3.5 ,

$$
w(\Gamma)-t(\Gamma)=2\left(n_{1}-2\right)
$$

where $n_{1}$ is the number of houses with connected boundary. Let $H_{2}$ be another special house of $\Gamma$. Since $H_{i}$ contains exactly one crossing, $d\left(H_{i}\right)=0$ for $i=1,2$ by Lemma 3.8. Moreover, since $\Gamma$ is saturated, $d(H)=2$ for any non-special house $H$ with connected boundary. Let $n$ be the number of such houses. Since $\partial H_{1}$ is disconnected, $n=n_{1}-1$ or $n=n_{1}$. Hence $n \geq n_{1}-1$, and

$$
w(\Gamma)-t(\Gamma)=2 n+d\left(H_{1}\right)+d\left(H_{2}\right)=2 n \geq 2\left(n_{1}-1\right) .
$$

This is a contradiction.

4. Rectangular rooms. In this section, we investigate a non-special house with connected boundary in a saturated minimal 4-chart.

Let $\Gamma$ be a chart, and $w$ a white vertex of $\Gamma$. In a small neighborhood of $w$, there are six short arcs. Let $\alpha_{1}, \alpha_{2}, \alpha_{3}$ be three consecutive arcs in this neighborhood. For $i=1,2,3$, let $B_{i}$ be the edge containing $\alpha_{i}$. Then $B_{1}$ and $B_{3}$ are called the side edges of $B_{2}$ at the white vertex $w$. 
Lemma 4.1. Let $\Gamma$ be a connected 4-chart, and $R$ a room of $\Gamma$. Let $\left\{g: D \rightarrow X_{R} ; \bar{A}_{1}, \ldots, \bar{A}_{n}\right\}$ be an associated set of $R$, and $A_{i}=g\left(\bar{A}_{i}\right)$ for each $i(1 \leq i \leq n)$. Suppose that $A_{1}, A_{3}$ are even labeled edges, and $A_{2}$ is odd labeled. If the room $R$ satisfies one of the following conditions, then we can reduce the number of white vertices of $\Gamma$ by $C$-moves.

(i) Neither $\left(A_{1}, A_{2}\right)$ nor $\left(A_{2}, A_{3}\right)$ is admissible.

(ii) $A_{2}$ does not contain distinguished arcs, and both $\left(A_{1}, A_{2}\right)$ and $\left(A_{2}, A_{3}\right)$ are admissible.

Proof. Suppose that $\Gamma$ satisfies (i). Then $A_{2}$ is not a loop. For $i=1,3$, let $B_{i}$ be the side edge of $A_{2}$ at the white vertex $A_{2} \cap A_{i}$ such that $B_{i} \neq A_{i}$. Then $B_{1}, A_{2}, B_{3}$ belong to the same room. Since $\left(A_{i}, A_{2}\right)$ is not admissible for $i=1,3,\left(B_{i}, A_{2}\right)$ is admissible. Hence $\left(B_{1}, B_{3}\right)$ is not admissible. The pair $\left(A_{1}, A_{3}\right)$ is not admissible. Apply a C-I-M2 move between $A_{1}$ and $A_{3}$, and a C-I-M2 move between $B_{1}$ and $B_{3}$. Then we can cancel the white vertices of $A_{2}$ by a C-I-M3 move. See Figure 4 .

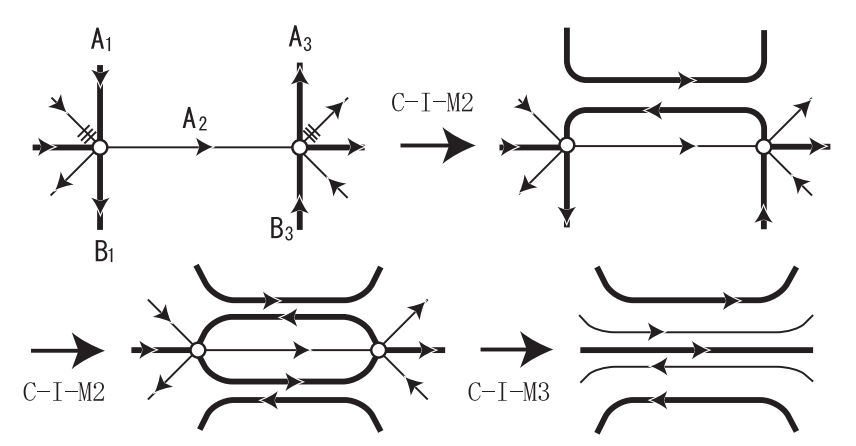

Fig. 4. The thick lines are edges of label 2. The other lines are edges of label 1 or 3 .

Suppose now that $\Gamma$ satisfies (ii). Since $A_{2}$ is not a distinguished edge, it is not a loop. For $i=1,3$, let $B_{i}$ be the side edge of $A_{2}$ at the white vertex $A_{2} \cap A_{i}$ such that $B_{i} \neq A_{i}$. Then $B_{1}, A_{2}, B_{3}$ belong to the same room. Since $A_{2}$ is not a distinguished edge and $\left(A_{i}, A_{2}\right)$ is admissible, $\left(B_{i}, A_{2}\right)$ is not admissible for $i=1,3$. Hence $\left(B_{1}, B_{3}\right)$ is not admissible. The pair $\left(A_{1}, A_{3}\right)$ is not admissible. Then we can cancel the white vertices of $A_{2}$ by C-I-M2 moves and a C-I-M3 move as above.

Lemma 4.2. Let $\Gamma$ be a connected minimal 4-chart, $H$ a house, and $B$ an odd labeled edge of $H$. Suppose that $H$ possesses no terminal edges of label 2 , and $B$ does not contain crossings and distinguished arcs. Let $E$ be a component of $H \backslash B$. If $\partial E \backslash B$ is connected, then $E$ possesses a distinguished arc or contains a crossing. 
Proof. Since $B$ does not contain crossings, it connects the boundary points of $X_{H}$. Let $\left\{g: D \rightarrow X_{E} ; \bar{A}_{1}, \ldots, \bar{A}_{n}\right\}$ be an associated set of $E$ such that $g\left(\bar{A}_{1}\right)=B$. Since $\partial E$ contains only one odd labeled edge $B, g\left(\bar{A}_{i}\right)$ is an even labeled edge for each $i=2, \ldots, n$.

Suppose that $E$ possesses neither distinguished arcs nor crossings. Take an outermost arc $\bar{B}^{\prime}$ in $D$ such that the odd labeled edge $g\left(\bar{B}^{\prime}\right)=B^{\prime}$ belongs to $E$. Then one of the components of $E \backslash B^{\prime}$ is a room with connected boundary, which possesses exactly one even labeled edge and one odd labeled edge, and which contains no distinguished arc. However, this contradicts Lemma 2.10.

Let $\Gamma$ be a connected 4-chart. A room $R$ of $\Gamma$ is rectangular if there exists an associated set of $R,\left\{g: D \rightarrow X_{R} ; \bar{A}_{1}, \bar{A}_{2}, \bar{A}_{3}, \bar{A}_{4}\right\}$, such that $g\left(\bar{A}_{1}\right), g\left(\bar{A}_{3}\right)$ are edges of label 2 , and $g\left(\bar{A}_{2}\right), g\left(\bar{A}_{4}\right)$ are edges of odd label.

Let $\Gamma$ be a connected 4 -chart, $R$ a room of $\Gamma$, and $\left\{g: D \rightarrow X_{R} ; \bar{P}_{1}, \ldots\right.$, $\left.\bar{P}_{n} ; \bar{A}_{1}, \ldots, \bar{A}_{n}\right\}$ an associated set of $R$. If $R$ satisfies one of the following conditions, then it is called an end room. See Figure 5.

(I) $n=5, g\left(\bar{A}_{1}\right)=g\left(\bar{A}_{5}\right)$ is a distinguished edge, $g\left(\bar{A}_{2}\right), g\left(\bar{A}_{4}\right)$ are edges of label 2 , and $g\left(\bar{A}_{3}\right)$ is an edge of odd label.

(II) $n=2, g\left(\bar{A}_{1}\right)$ is an edge of label 2 , and $g\left(\bar{A}_{2}\right)$ is a distinguished edge.

(III) $n=6, g\left(\bar{A}_{1}\right)=g\left(\bar{A}_{6}\right), g\left(\bar{A}_{3}\right)=g\left(\bar{A}_{4}\right)$ are distinguished edges, and $g\left(\bar{A}_{2}\right), g\left(\bar{A}_{5}\right)$ are edges of label 2 .

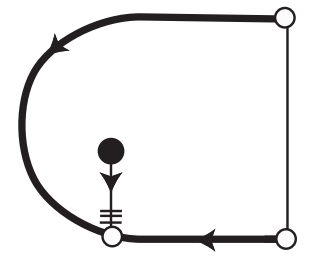

( I )

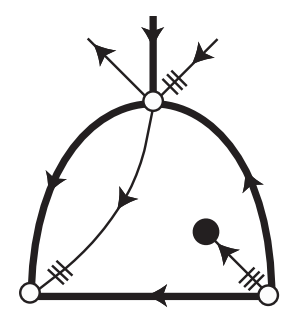

(IV)

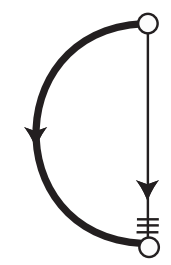

(II)

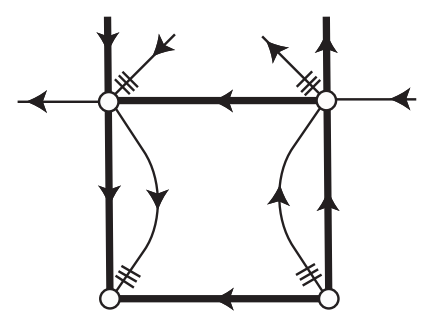

( $\mathrm{V})$

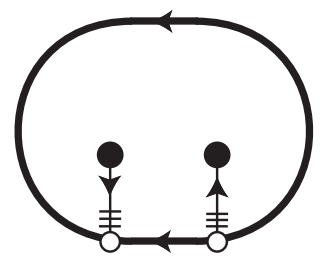

(III)

Fig. 5. The thick lines are edges of label 2. The other lines are edges of label 1 or 3 . 
The rooms satisfying (I), (II) and (III) are called end rooms of type (I), (II) and (III), respectively. Let $H$ be a house. If $H$ consists only of an end room of type (I) and an end room of type (II), then $H$ is called a house of type (IV). The house in Figure 5(IV) is an example. If $H$ consists only of two end rooms of type (II) and a rectangular room, then it is called a house of type $(\mathrm{V})$. An example is shown in Figure 5(V).

LEMma 4.3. Let $\Gamma$ be a saturated connected minimal 4-chart, and $H$ a non-special house of $\Gamma$. If the boundary of $H$ is connected, then $H$ satisfies one of the following two conditions:

(i) $H$ is an end room of type (III).

(ii) Two rooms in $H$, say $R, R^{\prime}$, are end rooms, and the other rooms, say $R_{1}, \ldots, R_{n}$, are rectangular. Moreover, we can renumber the rectangular rooms so that for each $i=0,1, \ldots, n, R_{i}$ and $R_{i+1}$ possess a common edge of odd label, where $R_{0}=R$ and $R_{n+1}=R^{\prime}$.

Let $A, A^{\prime}$ be edges of label 2 which belong to $H$. If $A, A^{\prime}$ belong to the same rectangular room, then $\left(A, A^{\prime}\right)$ is admissible.

Proof. Let $\left\{g: D \rightarrow X_{H} ; \bar{P}_{1}, \ldots, \bar{P}_{n} ; \bar{A}_{1}, \ldots, \bar{A}_{n}\right\}$ be an associated set of $H$. Since $\Gamma$ is saturated, $H$ possesses exactly two distinguished edges, say $B, B^{\prime}$. Since $\Gamma$ is minimal, $B \neq B^{\prime}$ by Remark 2.3(i). Since $H$ is non-special, it possesses no terminal edges of label 2 by Lemma 2.7. If $H$ does not possess other edges of odd label, then it is of type (III), (IV) or (V). Therefore we may assume that there exist other edges of odd label belonging to $H$, say $B_{1}, \ldots, B_{k}$. Let $\bar{B}_{i}, \bar{B}, \bar{B}^{\prime}$ be the $\operatorname{arcs}$ in $D$ with $g(\bar{B})=B, g\left(\bar{B}^{\prime}\right)=B^{\prime}$ and $g\left(\bar{B}_{i}\right)=B_{i}$ for all $i(1 \leq i \leq k)$. Since $B_{i}$ is not a distinguished edge, it is not terminal by Remark 2.3(ii). Hence it connects boundary points of $H$.

Suppose that $B$ or $B^{\prime}$ is terminal, say $B$ is a terminal edge containing the white vertex $g\left(\bar{P}_{1}\right)$. Let $\bar{P}^{\prime}$ be the point in $\partial D$ such that $B^{\prime}$ is a distinguished edge at the vertex $g\left(\bar{P}^{\prime}\right)$. Let $C_{1}, C_{2}$ be the components of $\partial D \backslash\left\{\bar{P}_{1}, \bar{P}^{\prime}\right\}$. By Lemma 4.2, each arc $\bar{B}_{i}(1 \leq i \leq k)$ connects a point in $C_{1}$ and a point in $C_{2}$. If not, then there exists a component $E$ of $H \backslash B_{i}$ possessing no distinguished arc and containing no crossing, and with $\partial E \backslash B_{i}$ connected. This contradicts Lemma 4.2. We may assume that the arc $\bar{B}_{i}$ connects $\bar{P}_{i+1}$ and $\bar{P}_{n-i-1}$ for $i=1, \ldots, k$. If $B^{\prime}$ is a terminal edge, then $n=2 k+6$, and $B^{\prime}$ has the white vertex $g\left(\bar{P}_{k+2}\right)$. See Figure 6(1). If $B^{\prime}$ is not terminal, then $n=2 k+5$, and $\bar{B}^{\prime}$ connects $\bar{P}_{k+2}$ and $\bar{P}_{k+3}$. See Figure 6(2).

Suppose that neither $B$ nor $B^{\prime}$ is terminal. We may assume that $\bar{B}$ connects $\bar{P}_{1}$ and $\bar{P}_{2}$. As above, we may assume that $\bar{B}_{i}$ connects $\bar{P}_{i+2}$ and $\bar{P}_{n-i+1}$ for $i=1, \ldots, k$. Since $B^{\prime}$ is not a terminal edge, it follows that $n=2 k+4$, and $\bar{B}^{\prime}$ connects $\bar{P}_{k+3}$ and $\bar{P}_{k+4}$. See Figure $6(3)$. 


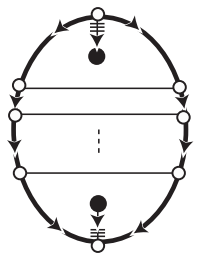

(1)

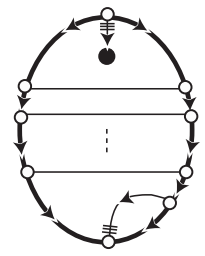

(2)

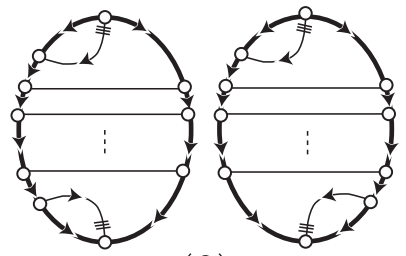

(3)

Fig. 6. The figures are preimages of associated sets. Hence two even labeled edges may be identified by the map $g$.

Let $\bar{P}, \bar{P}^{\prime}$ be the points of $\partial D$ such that $B, B^{\prime}$ are the distinguished edges at $g(\bar{P}), g\left(\bar{P}^{\prime}\right)$, respectively. Let $C_{1}, C_{2}$ be the components of $\partial D \backslash\left\{\bar{P}, \bar{P}^{\prime}\right\}$. Since no odd labeled edges except $B, B^{\prime}$ contain distinguished arcs, even labeled edges are oriented as shown in Figure 6 . That is, either both $C_{1}$ and $C_{2}$ are oriented from $\bar{P}$ to $\bar{P}^{\prime}$, or both from $\bar{P}^{\prime}$ to $\bar{P}$, where the orientations of $C_{1}$ and $C_{2}$ are induced from $\Gamma$. Hence we have the last statement in Lemma 4.3.

Lemma 4.4. Let $\Gamma$ be a saturated connected minimal 4-chart. Then any rectangular room of $\Gamma$ satisfies one of the following two conditions:

(i) There exists only one edge of label 2 of the room.

(ii) There exist two edges of label 2 of the room such that this pair of edges is admissible.

Proof. Let $R$ be a rectangular room of $\Gamma$. Let $\left\{g: D \rightarrow X_{R} ; \bar{A}_{1}, \bar{A}_{2}\right.$, $\left.\bar{A}_{3}, \bar{A}_{4}\right\}$ be an associated set of $R$ such that $g\left(\bar{A}_{1}\right)$ is an edge of label 2 . For $i=1,2,3,4$, set $A_{i}=g\left(\bar{A}_{i}\right)$. If $A_{1}=A_{3}$, then $R$ satisfies (i). Hence we may assume $A_{1} \neq A_{3}$. Suppose that $R$ possesses a distinguished arc. Since $\Gamma$ is saturated, $R$ is contained in a non-special house with connected boundary, say $H$. Since the two even labeled edges $A_{1}$ and $A_{3}$ belong to the rectangular room $R,\left(A_{1}, A_{3}\right)$ is admissible by Lemma 4.3. We may assume that $R$ possesses no distinguished arcs.

Suppose that $\left(A_{1}, A_{3}\right)$ is not admissible. Then there are two possibilities:

(i) $\left(A_{1}, A_{2}\right)$ or $\left(A_{1}, A_{4}\right)$ is not admissible.

(ii) $\left(A_{1}, A_{2}\right)$ and $\left(A_{1}, A_{4}\right)$ are admissible, and $A_{2}$ and $A_{4}$ do not contain distinguished arcs.

In case (i), if $\left(A_{1}, A_{2}\right)$ is not admissible, then neither is $\left(A_{2}, A_{3}\right)$. By Lemma 4.1(i), we can reduce the number of white vertices of $\Gamma$ by C-moves. This contradicts the minimality of $\Gamma$. Similarly, if $\left(A_{1}, A_{4}\right)$ is not admissible, then we also have a contradiction.

In case (ii), by Lemma 4.1(ii) we can reduce the number of white vertices of $\Gamma$ by C-moves. This contradicts the minimality of $\Gamma$. 
Lemma 4.5. Let $\Gamma$ be a saturated connected minimal 4-chart, and $H$ a non-special house. If the boundary of $H$ consists of two components, then $H$ consists of rectangular rooms $R_{1}, \ldots, R_{n}$ such that $R_{i}$ and $R_{i+1}$ possess a common edge of odd label for each $i=1, \ldots, n$, where $R_{n+1}=R_{1}$.

Proof. Let $C_{1}, C_{2}$ be the components of $\partial H$. Since $\Gamma$ is saturated, no odd labeled edge in $H$ is a distinguished edge. Since $H$ is a non-special house, it does not contain crossings. By Lemma 2.7, $H$ possesses no terminal edges of label 2. By using Lemma 4.2, we can show that any odd labeled edge in $H$ connects a point in $C_{1}$ and a point in $C_{2}$. Let $H^{\prime}$ be the complementary domain of $C_{k}$ with $H \subset H^{\prime}(k=1,2)$. Let $\left\{g: \bar{D} \rightarrow X_{H^{\prime}} ; A_{1}, \ldots, A_{n}\right\}$ be an associated set of $H^{\prime}$. Since $H$ possesses no terminal edge of label $2, g\left(\bar{A}_{i}\right) \neq$ $g\left(\bar{A}_{i+1}\right)$ for any $i$. Then the even labeled edges $g\left(\overline{A_{i}}\right), g\left(\bar{A}_{i+1}\right)$ have a common white vertex. Since $H$ possesses no distinguished arc, $\left(g\left(\bar{A}_{i}\right), g\left(\bar{A}_{i+1}\right)\right)$ is not admissible. Hence $\left(g\left(\bar{A}_{i}\right), g\left(\bar{A}_{j}\right)\right)$ is not admissible for all $i, j(1 \leq i \neq j \leq n)$, and $g\left(\bar{A}_{i}\right) \neq g\left(\bar{A}_{j}\right)$. Therefore each $C_{k}$ is a simple closed curve $(k=1,2)$, each room in $H$ is rectangular, and the closures of any two rooms have a common odd labeled edge or do not intersect.

Lemma 4.6. Let $\Gamma$ be a connected minimal 4-chart with exactly two crossings and with two special houses. Then each room in a non-special house is an end room or a rectangular room.

Proof. By Theorem 3.7, $\Gamma$ is saturated. By Lemma 3.5, the boundary of any non-special house is connected or has two components. Applying Lemmas 4.3 and 4.5 , we complete the proof.

5. Towns. Let $\Gamma$ be a connected 4-chart, $T$ a union of houses and rooms of $\Gamma$, and $X_{T}$ the closure of $T$. Let $w$ be a white vertex in $X_{T}$. There are six short arcs in a small neighborhood of $w$, say $\alpha_{1}, \alpha_{2}, \alpha_{3}, \alpha_{4}, \alpha_{5}, \alpha_{6}$. The white vertex $w$ is good if it satisfies the following condition: If $N \cap \Gamma$ contains at most three arcs of $\alpha_{i}(i=1, \ldots, 6)$, then no arc of $N \cap \Gamma$ is a distinguished arc. Here, $N$ is a small neighborhood of $w$ in $X_{T}$. We denote the number of $\operatorname{arcs} \alpha_{i}$ lying in $N \cap \Gamma$ by $n(w, T)$.

We call $T$ a town if:

(i) The closure $X_{T}$ of $T$ is a disjoint union of disks.

(ii) Either all even labeled edges in $\partial X_{T}$ are oriented clockwise, or all are oriented anticlockwise.

(iii) Any white vertex in $X_{T}$ is good.

A town $T$ is good if the following holds: Let $H$ be a house of type (IV) in $X_{T}$. Let $B$ be the even labeled edge such that $B$ belongs to $H$ and both odd labeled edges in $H$ are distinguished edges at the vertices of $B$. If all even labeled edges in $\partial X_{T}$ are oriented clockwise (resp. anticlockwise), then $B$ 
is oriented anticlockwise in $\partial X_{H}$ (resp. clockwise), where $X_{H}$ is the closure of $H$.

Let $\Gamma$ be a connected 4-chart, $T$ a town, $X_{T}$ the closure of $T, B$ an even labeled edge in $\partial X_{T}$, and $B_{1}, B_{2}$ two odd labeled edges with $B_{i} \not \subset X_{T}$ for $i=1,2$. The triplet $\left(B_{1}, B, B_{2}\right)$ is a semi-repeat triplet with respect to $T$ if it satisfies the following three conditions:

(i) The three edges belong to the same room $R$.

(ii) There exists an associated set $\left\{g: D \rightarrow X_{R} ; \bar{A}_{1}, \ldots, \bar{A}_{n}\right\}$ of $R$ such that $g\left(\bar{A}_{1}\right)=B_{1}, g\left(\bar{A}_{2}\right)=B$, and $g\left(\bar{A}_{3}\right)=B_{2}$.

(iii) The label of $B_{1}$ is different from that of $B_{2}$.

Moreover, if both $B_{1}$ and $B_{2}$ are distinguished edges at the vertices of $B$, then $\left(B_{1}, B, B_{2}\right)$ is called a repeat triplet with respect to $T$.

Definition 5.1. Let $\Gamma$ be a connected 4-chart, $T$ a town, and $\left(B_{1}, B, B_{2}\right)$ a semi-repeat triplet with respect to $T$. The triplet $\left(B_{1}, B, B_{2}\right)$ is good if it satisfies one of the following two conditions:

(a) For $i=1,2$, if $\left(B_{i}, B\right)$ is not admissible, then $B_{i}$ is not a distinguished edge.

(b) If neither $B_{1}$ nor $B_{2}$ is a distinguished edge at a vertex of $B$, then $B_{1}, B_{2}, B$ belong to the same rectangular room, and $\left(B_{i}, B\right)$ is admissible for $i=1,2$.

Lemma 5.2. Let $\Gamma$ be a saturated connected minimal 4-chart, $T$ a town, and $\left(B_{1}, B, B_{2}\right)$ a repeat triplet with respect to $T$. Let $H$ be the house which possesses $B_{1}$ and $B_{2}$. Then $T \cup H$ is a town, and $X_{T} \cap X_{H}=B$, where $X_{T}, X_{H}$ are the closures of $T, H$ respectively. Moreover, if $T$ is good, then so is $T \cup H$.

Proof. Since $B_{1}$ and $B_{2}$ each contain distinguished arcs, $H$ possesses distinguished arcs. Since $\Gamma$ is saturated, $H$ is a non-special house with connected boundary. By Lemma 4.3,

(i) if $B_{1}, B_{2}$ are terminal edges, then $H$ is of type (III),

(ii) if only one of $B_{1}, B_{2}$ is terminal, then $H$ is of type (IV), and

(iii) if neither $B_{1}$ nor $B_{2}$ is terminal, then $H$ is of type (V).

See Figure 5(III), (IV) and (V). Let $B^{\prime}$ be an edge of label 2 in $\partial X_{H}$ different from $B$. Since $T$ is a town, $B^{\prime}$ is not contained in $X_{T}$. Let $w$ be a white vertex of $X_{H}$, and $N$ a small neighborhood of $w$ in the closure of $T \cup X$. We prove that $N$ is a disk. There are two cases: (a) $w \in B$, (b) $w \notin B$.

In case (a), $n(w, H)=3$ and $n(w, T \cup H) \geq 4$. If $N$ is not a disk, then $N$ is a cone over a disjoint union of two arcs, and then a neighborhood of $w$ in $X_{T}$ is a cone over a disjoint union of two arcs. This contradicts $X_{T}$ being 
a disjoint union of disks. Hence $N$ is a disk, and $w$ is a good vertex with respect to $T \cup H$.

In case (b), if $w \notin \partial X_{T}$, then $N$ is a disk. Suppose that $w \in \partial X_{T}$ and $N$ is not a disk. Since $n(w, H)=3$, we have $n(w, T) \leq 3$. Since a small neighborhood of $w$ in $X_{T}$ does not contain distinguished arcs, $n(w, T)=2$. We see that there exists an even labeled edge $C$ containing the white vertex $w$ and contained in $\partial X_{T}$ such that $C$ is oriented clockwise (resp. anticlockwise) if the edge $B$ in $\partial X_{T}$ is oriented anticlockwise (resp. clockwise). However, this contradicts $T$ being a town. Thus $w \notin \partial X_{T}$. We have $n(w, T \cup H)=3$ and $w$ is a good white vertex with respect to $T \cup H$, and $N$ is a disk. Moreover, $X_{T} \cap X_{H}=B$, and the closure of $T \cup H$ is a disjoint union of disks. Therefore $T \cup H$ is a town.

Suppose that $H$ is a house of type (IV). Let $B$ be the even labeled edge such that $B$ belongs to $H$ and both odd labeled edges in $H$ are distinguished edges at the vertices of $B$. If all even labeled edges in $\partial X_{T}$ are oriented clockwise (resp. anticlockwise), then all even labeled edges in the boundary of the closure of $T \cup H$ are oriented clockwise (resp. anticlockwise), and then $B$ is oriented anticlockwise (resp. clockwise) in $\partial X_{H}$. If $T$ is good, then the other house of type (IV) satisfies the same condition. Therefore if $T$ is good, then so is $T \cup H$.

LEMMA 5.3. Let $\Gamma$ be a saturated connected minimal 4-chart, $T$ a town, and $\left(B_{1}, B, B_{2}\right)$ a good semi-repeat triplet with respect to $T$ such that $B_{1}$ is a distinguished edge at a vertex of $B$, but $B_{2}$ is not. Let $R$ be the union of all rooms which possess $B_{1}$. Then $T \cup R$ is a town, and $X_{T} \cap X_{R}=B$, where $X_{T}, X_{R}$ are the closures of $T, R$ respectively. Moreover, if $T$ is good, then so is $T \cup R$.

Proof. Since $\Gamma$ is minimal, $B_{2}$ is not a terminal edge. Since $B_{1}$ contains a distinguished arc, $R$ possesses a distinguished arc. Since $\Gamma$ is saturated, $R$ is contained in a non-special house with connected boundary. By Lemma 4.3,

(i) if $B_{1}$ is a terminal edge, then $R$ is an end room of type (I),

(ii) if neither $B_{1}$ nor $B_{2}$ is terminal, then there exist an end room $R_{1}$ of type (II) and a rectangular room $R_{2}$ with $R=R_{1} \cup R_{2}$.

In case (i), let $B^{\prime}$ be another even labeled edge in $R$. In case (ii), let $B^{\prime}$ be another even labeled edge in $R_{2}$. If $\left(B, B_{2}\right)$ is admissible, then $\left(B^{\prime}, B_{2}\right)$ is not by Lemma 4.3. Hence the arcs in a small neighborhood of $B_{2}$ are oriented as shown in Figure $7(\mathrm{a})$. If $\left(B, B_{2}\right)$ is not admissible, then $B_{2}$ is not a distinguished edge, because $\left(B_{1}, B, B_{2}\right)$ is good. Hence the arcs in a small neighborhood of $B_{2}$ are oriented as shown in Figure $7(\mathrm{~b})$.

Let $B^{\prime \prime}$ be an edge of label 2 in $\partial X_{R}$ different from $B$. Since $T$ is a town, $B^{\prime \prime}$ is not contained in $X_{T}$. Let $w$ be a white vertex in $X_{R}$, and $N$ a small 
(a)

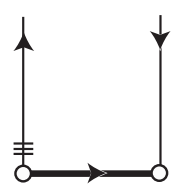

(b)

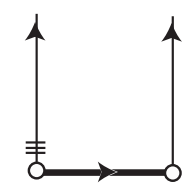

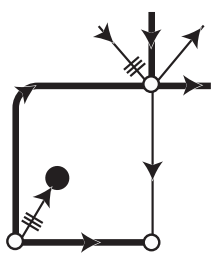
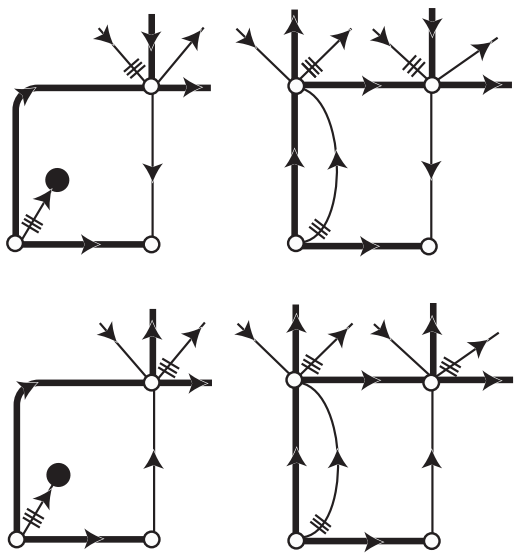

Fig. 7. The thick lines are edges of label 2. The other lines are edges of label 1 or 3 .

neighborhood of $w$ in the closure of $T \cup R$. We prove that $N$ is a disk. For $n(w, R)=3$ or $w \in B$, this can be shown in a similar way to Lemma 5.2. Hence we may assume that $n(w, R)=2, w \notin B$, and $N$ is not a disk. If $w \notin \partial X_{T}$, then $N$ is a disk, so assume $w \in \partial X_{T}$.

If $n(w, T)=4$, then there exists an even labeled edge $C$ containing the white vertex $w$ and contained in $\partial X_{T}$ such that $C$ is oriented clockwise (resp. anticlockwise) if the edge $B$ in $\partial X_{T}$ is oriented anticlockwise (resp. clockwise). However, this contradicts $T$ being town.

If $n(w, T)=2$ or 3 , then $N$ contains no distinguished arcs, because $w$ is a good vertex with respect to $T$. We see that there exists an even labeled edge $C$ containing the white vertex $w$ and contained in $\partial X_{T}$ such that $C$ is oriented clockwise (resp. anticlockwise) if the edge $B$ in $\partial X_{T}$ is oriented anticlockwise (resp. clockwise). However, this contradicts $T$ being town.

Hence $w \notin \partial X_{T}, n(w, T \cup R)=n(w, R)=2, N$ is a disk, and $w$ is a good white vertex with respect to $T \cup R$. Moreover, $X_{T} \cap X_{R}=B$, and the closure of $T \cup R$ is a disjoint union of disks. Therefore $T \cup R$ is a town.

Lemma 5.4. Let $\Gamma$ be a saturated connected minimal 4-chart, $T$ a town, and $\left(B_{1}, B, B_{2}\right)$ a good semi-repeat triplet with respect to $T$ such that neither $B_{1}$ nor $B_{2}$ is a distinguished edge at a vertex of $B$. Let $R$ be the rectangular room which possesses $B_{1}$ and $B_{2}$. Then $T \cup R$ is a town, $X_{T} \cap X_{R}=B$, where $X_{T}, X_{R}$ are the closures of $T, R$ respectively, and there exists a repeat triple with respect to $T \cup R$. Moreover, if $T$ is good, then so is $T \cup R$.

Proof. Let $B^{\prime}$ be another labeled edge which belongs to $R$. Then $\left(B, B^{\prime}\right)$ is admissible by Lemma 4.4. The proof is now similar to that of Lemma 5.3. 
Lemma 5.5 ([3, Remarks 8(2)]). Let $\Gamma$ be an $n$-chart, $m$ the number of black vertices of $\Gamma$, and $F$ the closure of the surface braid obtained from $\Gamma$. Then $\chi(F)=2 n-m$.

To show Theorem 1.1, it suffices to prove that any saturated minimal 4-chart with exactly two crossings has at least eight black vertices. Let $T$ be a town. Denote by $b(T)$ the number of black vertices in $X_{T}$, where $X_{T}$ is the closure of $T$. If a black vertex $w$ is contained in a house of type (IV), then the weight of $w$ is defined to be 1 . Otherwise it is $1 / 2$. Denote by $b^{\prime}(T)$ the sum of the weights of all black vertices in $X_{T}$.

Lemma 5.6. Let $T_{1}, T_{2}$ be good towns such that all even labeled edges in $\partial X_{T_{1}}$ (resp. $\left.\partial X_{T_{2}}\right)$ are oriented clockwise (resp. anticlockwise). Then $b\left(T_{1} \cup T_{2}\right) \geq b^{\prime}\left(T_{1}\right)+b^{\prime}\left(T_{2}\right)$.

Proof. Let $H$ be a house of type (IV) with $H \subset T_{1} \cup T_{2}$. Let $B$ be an even labeled edge in $H$ such that both odd labeled edges in $H$ are distinguished edges at the vertices of $B$. Since $T_{1}$ and $T_{2}$ are good towns, if $B$ is oriented clockwise (resp. anticlockwise) in $\partial H$, then $H$ is contained in $T_{2}$ (resp. $T_{1}$ ). Hence no house of type (IV) in $T_{i}$ intersects $T_{j},\{i, j\}=\{1,2\}$. Thus no black vertex of weight 1 in $T_{i}$ belongs to $T_{j}$. Therefore $b\left(T_{1} \cup T_{2}\right) \geq b^{\prime}\left(T_{1}\right)+b^{\prime}\left(T_{2}\right)$.

Lemma 5.7. Let $\Gamma$ be a saturated connected minimal 4-chart, $T$ a town, and $\left(B_{1}, B, B_{2}\right)$ a repeat triplet with respect to $T$. Then there exists a town $T^{\prime}$ such that $b\left(T^{\prime}\right) \geq b(T)+1, b^{\prime}\left(T^{\prime}\right)=b^{\prime}(T)+1, T^{\prime} \supset T$, and $X_{T} \cap X=B$, where $X_{T}, X$ are the closures of $T, T^{\prime} \backslash T$ respectively. Moreover, if $T$ is good, then so is $T^{\prime}$.

Proof. Let $H_{1}$ be the house which possesses $B_{1}$ and $B_{2}$, and $X_{H_{1}}$ its closure. Then we have the three possibilities (i), (ii) and (iii) as in the proof of Lemma 5.2. By Lemma 5.2, $T \cup H_{1}$ is a town, and $X_{T} \cap X_{H_{1}}=B$. In cases (i) and (ii), $T \cup H_{1}$ has more black vertices than $T$, and $b^{\prime}\left(T \cup H_{1}\right)=b^{\prime}(T)+1$. In case (iii), the orientations of all edges in a neighborhood of $X_{H_{1}}$ are as in Figure $5(\mathrm{~V})$. Hence there exists a repeat triplet with respect to $T \cup H_{1}$. Let $T_{0}=T, T_{1}=T \cup H_{1}$, and $\left(B_{1,1}, A_{1}, B_{1,2}\right)=\left(B_{1}, B, B_{2}\right)$. To repeat this argument, there exists a repeat triplet $\left(B_{i, 1}, A_{i}, B_{i, 2}\right)$ with respect to $T_{i-1}$ $(i=2, \ldots, n)$ and there exists a town $T_{i}(i=2, \ldots, n)$ such that

(a) $T_{i}$ is the union of $T_{i-1}$ and a house $H_{i}$ which possesses $B_{i, 1}$ and $B_{i, 2}$ for $i=1, \ldots, n$,

(b) $B_{n, 1}$ or $B_{n, 2}$ is a terminal edge,

(c) $X_{T_{i-1}} \cap X_{H_{i}}=A_{i}$ for $i=1, \ldots, n$,

where $X_{T_{i}}$ and $X_{H_{i}}$ are the closures of $T_{i}$ and $H_{i}$ respectively. See Figure 8. By Lemma 5.2, $T_{n}$ is the desired town. 


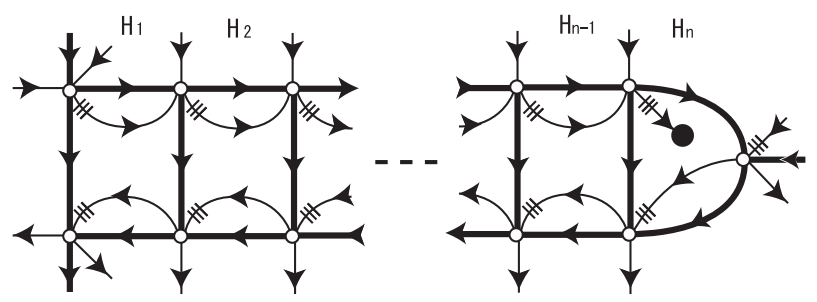

Fig. 8. The thick lines are edges of label 2. The other lines are edges of label 1 or 3 .

The set $H_{1} \cup \cdots \cup H_{n}$ is called the tower with respect to $\left(B_{1}, B, B_{2}\right)$, and the house $H_{n}$ is called the top house of this tower.

Lemma 5.8. Let $\Gamma$ be a saturated connected minimal 4-chart, and $T$ a town. Let $\left(B_{1}, B, B_{2}\right)$ be a good semi-repeat triplet with respect to $T$ such that neither $B_{1}$ nor $B_{2}$ is a distinguished edge at a vertex of $B$. Then there exists a town $T^{\prime}$ such that $b\left(T^{\prime}\right) \geq b(T)+1, b^{\prime}\left(T^{\prime}\right)=b^{\prime}(T)+1, T^{\prime} \supset T$, and $X_{T} \cap X=B$, where $X_{T}, X$ are the closures of $T, T^{\prime} \backslash T$ respectively. Moreover, if $T$ is good, then so is $T^{\prime}$.

Proof. By Definition 5.1(b), $\left(B_{1}, B\right)$ and $\left(B_{2}, B\right)$ are admissible. Let $R$ be the rectangular room which possesses $B_{1}$ and $B_{2}, B^{\prime}$ the even labeled edge in $R$ with $B \neq B^{\prime}$, and $C_{i}$ the side edge of $B^{\prime}$ with $C_{i} \neq B_{i}$ for $i=1,2$. By Lemma $4.4,\left(B, B^{\prime}\right)$ is admissible. The pair $\left(C_{i}, B^{\prime}\right)$ is not admissible, and $C_{i}$ is a distinguished edge at the vertex of $B^{\prime}$ for $i=1,2$. By Lemma 5.4, $T \cup R$ is a town. The triplet $\left(C_{1}, B^{\prime}, C_{2}\right)$ is a repeat triplet with respect to the town $T \cup R$. Hence we have the desired town by Lemma 5.7.

6. A special house with connected boundary. In this section, we investigate a special house with connected boundary.

Lemma 6.1. Let $\Gamma$ be a saturated connected minimal 4-chart, and $H$ a special house with connected boundary. If $H$ contains exactly one crossing, then there exist only four even labeled edges $A_{1}, A_{2}, A_{3}, A_{4}$ and only four odd labeled edges $B_{1}, B_{2}, B_{3}, B_{4}$ such that

(i) $B_{i} \cap B_{j}$ is a crossing for $i \neq j$,

(ii) $A_{i} \cap A_{i+1}$ is a white vertex $w_{i}$ for $i=1,2,3,4$,

(iii) $A_{i} \cap B_{i}=\left\{w_{i}\right\}$ for $i=1,2,3,4$, where $A_{5}=A_{1}$. That is, $H$ is as illustrated in Figure 9.

Proof. Let $B$ be an edge containing a crossing which belongs to $H$. Since $H$ contains exactly one crossing, the other vertex of $B$ is white. There exist four such edges of odd label. By Lemma 3.8, $H$ possesses no terminal edges of label 2 . 


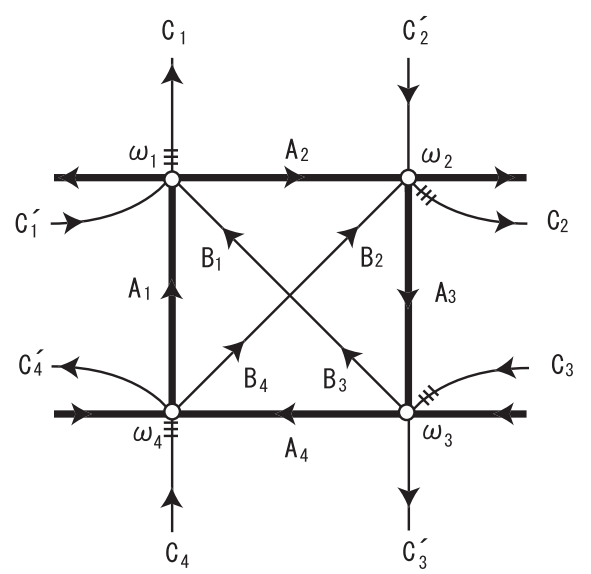

Fig. 9

Suppose that an odd labeled edge $B^{\prime}$ belongs to $H$ and contains no crossings. Since $\Gamma$ is saturated, $H$ possesses no distinguished edges and $B^{\prime}$ is not a distinguished edge. By Lemma 4.2, each component of $H \backslash B^{\prime}$ contains a crossing. However, this contradicts the assumption that $H$ contains exactly one crossing. Hence no such odd labeled edge $B^{\prime}$ exists. Therefore there exist only four edges of label 2 and only four edges of odd label satisfying the conditions in Lemma 6.1.

Let $\Gamma, H, A_{i}, B_{i}, w_{i}$ be as above $(i=1,2,3,4)$. We may assume that $\left(B_{1}, B_{2}\right),\left(A_{1}, B_{1}\right)$ are admissible, For $i=1,2,3,4$, let $C_{i}, C_{i}^{\prime}$ be the odd labeled edges containing the white vertex $w_{i}$ such that $C_{i}$ is a distinguished edge at $w_{i}$ and $C_{i}^{\prime} \neq B_{i}$. Then $C_{1}, A_{2}, C_{2}^{\prime}$ belong to the same room, and so do $C_{2}, A_{3}, C_{3}$ and $C_{3}^{\prime}, A_{4}, C_{4}$ and $C_{1}^{\prime}, A_{1}, C_{4}^{\prime}$.

Lemma 6.2. Let $\Gamma, H, A_{i}, B_{i}, C_{i}, C_{i}^{\prime}$, $w_{i}$ be as above $(i=1,2,3,4)$. Then:

(i) $H$ is a good town.

(ii) $\left(C_{2}, A_{3}, C_{3}\right)$ is a repeat triplet with respect to $H$.

(iii) $\left(C_{1}, A_{2}, C_{2}^{\prime}\right)$ and $\left(C_{3}^{\prime}, A_{4}, C_{4}\right)$ are good semi-repeat triplets with respect to $H$.

(iv) If $\Gamma$ has exactly two crossings, then $\left(C_{1}^{\prime}, A_{1}, C_{4}^{\prime}\right)$ is a good semirepeat triplet with respect to $H$.

(v) $C_{1}$ or $C_{4}$ is not a terminal edge.

Proof. We first show (iv). Suppose that $\Gamma$ has exactly two crossings. Since $H$ contains only one crossing, $\Gamma$ has two special houses. If $C_{1}^{\prime}$ and $C_{4}^{\prime}$ belong to a non-special house, then $C_{1}^{\prime}, A_{1}, C_{4}^{\prime}$ belong to a rectangular room by Lemma 4.6. The pairs $\left(C_{1}^{\prime}, A_{1}\right)$ and $\left(C_{4}^{\prime}, A_{1}\right)$ are admissible. Hence $\left(C_{1}^{\prime}, A_{1}, C_{4}^{\prime}\right)$ is a good semi-repeat triplet with respect to $H$. If $C_{1}^{\prime}$ and $C_{4}^{\prime}$ belong to a special house, say $H^{\prime}$, then $H^{\prime}$ contains exactly one crossing, 
and then $\partial H^{\prime}$ is connected by Lemma 3.9. By Lemma $6.1, H$ and $H^{\prime}$ are houses as shown in Figure 10.

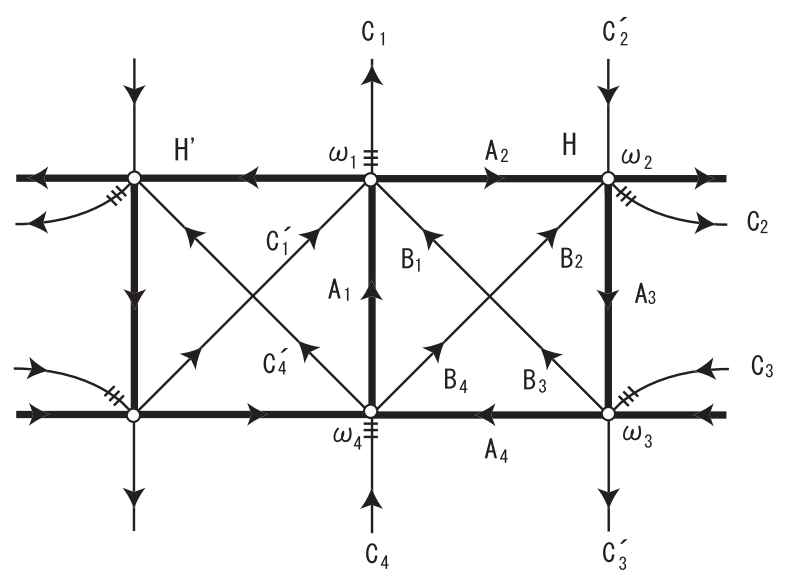

Fig. 10

Let $A$ be the even labeled edge containing the white vertex $w_{4}$ different from $A_{1}$ and $A_{4}$, and $w$ the white vertex of $A$ with $w \neq w_{4}$. Let $B$ be the odd labeled edge containing $w$ such that $B$ is not a distinguished edge at $w$, and $H^{\prime} \cap B=\emptyset$. Since $\Gamma$ is saturated, $B, C_{4}, C_{3}^{\prime}$ belong to the same non-special house with connected boundary. By Lemma $4.3, C_{4}$ is a terminal edge or $C_{4}=B$ or $C_{4}=C_{3}^{\prime}$. Since the label of $C_{4}$ is different from those of $C_{3}^{\prime}$ and $B, C_{4}$ is a terminal edge and $B=C_{3}^{\prime}$. However, $B$ is oriented from $w$ to the other white vertex, and $C_{3}^{\prime}$ is oriented from $w_{3}$ to the other white vertex. This is a contradiction. Therefore $C_{1}^{\prime}$ and $C_{4}^{\prime}$ belong to a non-special house, and $\left(C_{1}^{\prime}, A_{1}, C_{4}^{\prime}\right)$ is a good semi-repeat triplet with respect to $H$.

We now show (v) by means of Figure 11. Let $W$ be the tower with respect to $\left(C_{2}, A_{3}, C_{3}\right)$, and $H^{\prime}$ the top house of $W$. Suppose that $C_{1}$ and $C_{4}$ are both terminal edges. In general, for any two charts, if there exists a disk $E$ such that $\partial E$ intersects both charts transversally or intersects neither, and if the charts do not have black vertices on $E$ and coincide in the complement of $E$, then one chart is C-move equivalent to the other (see [3, Lemma 16]). Let $E$ be a neighborhood of the closure of $(H \cup W) \backslash H^{\prime}$. The second figure in Figure 11 can be obtained from the first by applying this move to the disk $E$. The third figure can be obtained from the second by C-II moves. The fourth figure can be obtained from third by a C-III-1 move. The final figure can be obtained from the fourth by a C-II move. Hence one white vertex can be cancelled and the number of crossings does not change. This contradicts the minimality of $\Gamma$. 

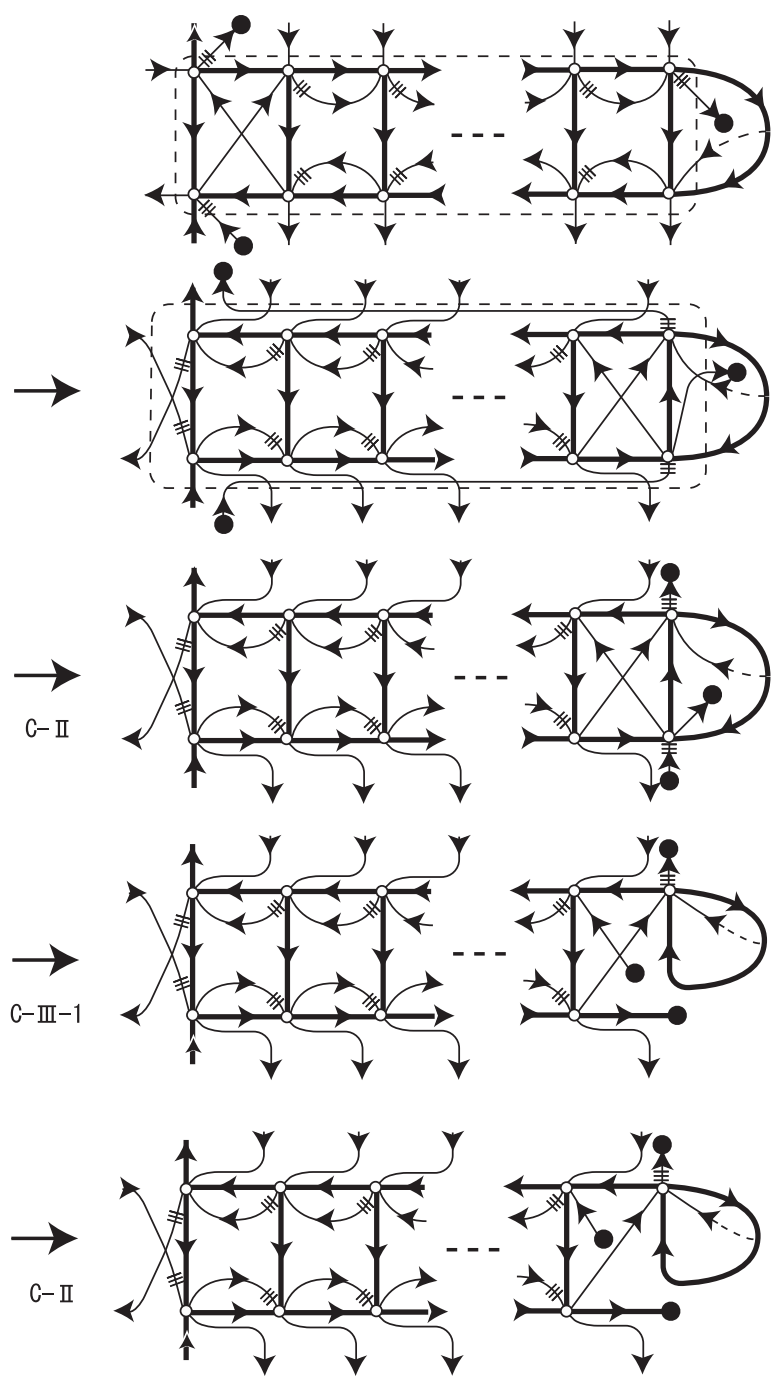

Fig. 11

Lemma 6.3. Let $\Gamma, H, A_{i}, B_{i}, C_{i}, C_{i}^{\prime}, w_{i}$ be as above $(i=1,2,3,4)$, and $T$ a town such that $X_{T} \cap X_{H}=\emptyset$ and $T \cup H$ is a good town. Suppose that $\Gamma$ has exactly two crossings. If neither $C_{1}$ nor $C_{4}$ is a terminal edge, then there exists a good town $T^{\prime}$ with $H \subset T^{\prime}, X_{T} \cap X_{T^{\prime}}=\emptyset, b\left(T^{\prime}\right) \geq 4$, and $b^{\prime}\left(T^{\prime}\right)=4$. Here, $X_{T}, X_{H}, X_{T^{\prime}}$ are the closures of $T, H, T^{\prime}$.

Proof. By Lemma 6.2(ii), $\left(C_{2}, A_{3}, C_{3}\right)$ is a repeat triplet with respect to $H$. Let $R_{i}$ be the union of all rooms which possess $C_{i}$ for $i=1,4$. By Lemma 6.2(iii), $\left(C_{1}, A_{2}, C_{2}^{\prime}\right)$ and $\left(C_{3}^{\prime}, A_{4}, C_{4}\right)$ are good semi-repeat triplets with respect to $H$. By Lemma 5.3, $H \cup R_{1} \cup R_{4}$ is a good town as shown 


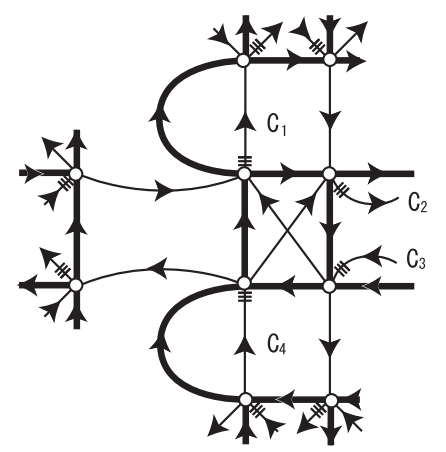

Fig. 12

in Figure 12. Let $A_{1}^{\prime}, A_{4}^{\prime}$ be the even labeled edges such that $C_{i}, A_{i}^{\prime}$ belong to the same rectangular room for $i=1,4, A_{1}^{\prime} \neq A_{2}$ and $A_{4}^{\prime} \neq A_{4}$. For $i=1,4$, let $v_{i}, v_{i}^{\prime}$ be the white vertices of $A_{i}^{\prime}$, and $E_{i}, E_{i}^{\prime}$ the distinguished edges at $v_{i}, v_{i}^{\prime}$ respectively. Then $\left(E_{i}, A_{i}^{\prime}, E_{i}^{\prime}\right)$ is a repeat triplet with respect to $H \cup R_{1} \cup R_{4}$ for $i=1,4$. Hence there are three repeat triplets with respect to $H \cup R_{1} \cup R_{4}$. Let $W_{1}, W_{2}, W_{3}$ be the towers with respect to $\left(C_{2}, A_{3}, C_{3}\right),\left(E_{1}, A_{1}^{\prime}, E_{1}^{\prime}\right),\left(E_{4}, A_{4}^{\prime}, E_{4}^{\prime}\right)$ respectively. Let

$$
T_{1}=H \cup R_{1} \cup R_{4} \cup W_{1} \cup W_{2} \cup W_{3} .
$$

By Lemma 5.7, $T_{1}$ is a good town, $b\left(T_{1}\right) \geq 3, b^{\prime}\left(T_{1}\right)=3, X_{T} \cap X_{T_{1}}=\emptyset$, and $T \cup T_{1}$ is a good town, where $X_{T_{1}}$ is the closure of $T_{1}$. Since $\Gamma$ has exactly two crossings, by Lemma $6.2(\mathrm{iv}),\left(C_{1}^{\prime}, A_{1}, C_{4}^{\prime}\right)$ is a good semi-repeat triplet for $H$. We see that $\left(C_{1}^{\prime}, A_{1}, C_{4}^{\prime}\right)$ is a good semi-repeat triplet for $T_{1}$. By Lemma 5.8, there exists a good town $T_{2}$ such that $H \subset T_{1} \subset T_{2}, b\left(T_{2}\right) \geq 4$, $b^{\prime}\left(T_{2}\right)=4, X_{T} \cap X_{T_{2}}=\emptyset$ and $T \cup T_{2}$ is a good town, where $X_{T_{2}}$ is the closure of $T_{2}$.

Lemma 6.4. Let $\Gamma, H, A_{i}, B_{i}, C_{i}, C_{i}^{\prime}, w_{i}$ be as above $(i=1,2,3,4)$, and $T$ a good town such that $X_{T} \cap X_{H}=\emptyset$ and $T \cup H$ is a good town. Suppose that $\Gamma$ has exactly two crossings. If $C_{1}$ or $C_{4}$ is a terminal edge, then there exists a good town $T^{\prime}$ with $H \subset T^{\prime}, X_{T} \cap X_{T^{\prime}}=\emptyset, b\left(T^{\prime}\right) \geq 4$, and $b^{\prime}\left(T^{\prime}\right)=3+1 / 2$. Here, $X_{T}, X_{H}, X_{T^{\prime}}$ are the closures of $T, H, T^{\prime}$.

Proof. By Lemma 6.2(v), we may assume that only one of $C_{1}$ and $C_{4}$, say $C_{4}$, is a terminal edge. Since $\Gamma$ is saturated, $C_{4}$ belongs to a non-special house with connected boundary. By Lemma 4.3, $C_{4}$ belongs to an end room of type (I), and the weight of the black vertex of $C_{4}$ is $1 / 2$. For $i=1,4$ let $R_{i}$ be the union of all rooms which possess $C_{i}$. By Lemma 5.3, $H \cup R_{1} \cup R_{4}$ is a good town as shown in Figure 13 and there are two repeat triplets with respect to $H \cup R_{1} \cup R_{4}$. Since $\Gamma$ has exactly two crossings, $\left(C_{1}^{\prime}, A_{1}, C_{4}^{\prime}\right)$ is a good semi-repeat triplet for $H$ by Lemma 6.2(iv). We conclude that 


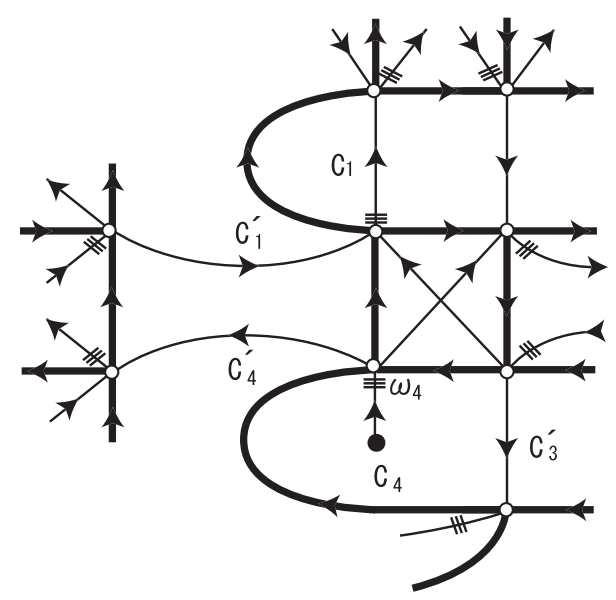

Fig. 13

$\left(C_{1}^{\prime}, A_{1}, C_{4}^{\prime}\right)$ is a good semi-repeat triplet for $H \cup R_{1} \cup R_{4}$. In a similar way to Lemma 6.3, there exists a good town $T_{1}$ such that $H \cup R_{1} \cup R_{4} \subset T_{1}$, $b\left(T_{1}\right) \geq 4, b^{\prime}\left(T_{1}\right)=3+1 / 2, X_{T} \cap X_{T_{1}}=\emptyset$ and $T \cup T_{1}$ is a good town, where $X_{T_{1}}$ is the closure of $T_{1}$.

\section{Proof of the main theorem}

THEOREM 7.1. Let $\Gamma$ be a connected minimal 4-chart with exactly two crossings and two special houses $H_{1}, H_{2}$. Then $\Gamma$ contains at least eight black vertices.

Proof. By Theorem 3.7, $\Gamma$ is saturated. Suppose that $\partial H_{1}$ and $\partial H_{2}$ are both oriented clockwise or both anticlockwise. Then $H_{1} \cup H_{2}, H_{1}, H_{2}$ are good towns. By Lemma 6.2, each $H_{i}$ satisfies one of the conditions in Lemmas 6.3 and 6.4. Hence for each $i$ there exists a good town $T_{i}$ with $H_{i} \subset T_{i}$ and $b\left(T_{i}\right) \geq 4$. Moreover $T_{1} \cap T_{2}=\emptyset$. Hence $b\left(T_{1} \cup T_{2}\right) \geq 8$.

Suppose that the orientation of one of $H_{1}$ and $H_{2}$ is clockwise, and of the other anticlockwise. By Lemmas 6.3 and 6.4, for each $i=1,2$ there exists a good town $T_{i}$ with $H_{i} \subset T_{i}$ and $b^{\prime}\left(T_{i}\right)=3+1 / 2$. Lemma 5.6 yields $b\left(T_{1} \cup T_{2}\right) \geq b^{\prime}\left(T_{1}\right)+b^{\prime}\left(T_{2}\right)=7$, which completes the proof since the number of black vertices of $\Gamma$ is even.

Proof of Theorem 1.1. We may assume that $\Gamma$ is a connected minimal saturated 4-chart with exactly two crossings by the Main Theorem in [4]. Suppose that the closure of the surface braid obtained from $\Gamma$ is one 2sphere. Since $\Gamma$ is a 4-chart, it has exactly six black vertices by Lemma 5.5. If $\Gamma$ has only one special house, then it contains at least eight black vertices by Lemma 3.2. If $\Gamma$ has two special houses, then it contains at least eight 
black vertices by Theorem 7.1. This contradicts $\Gamma$ having exactly six black vertices.

Finally, in Figure 14 we exhibit a saturated 4-chart with eight black vertices.

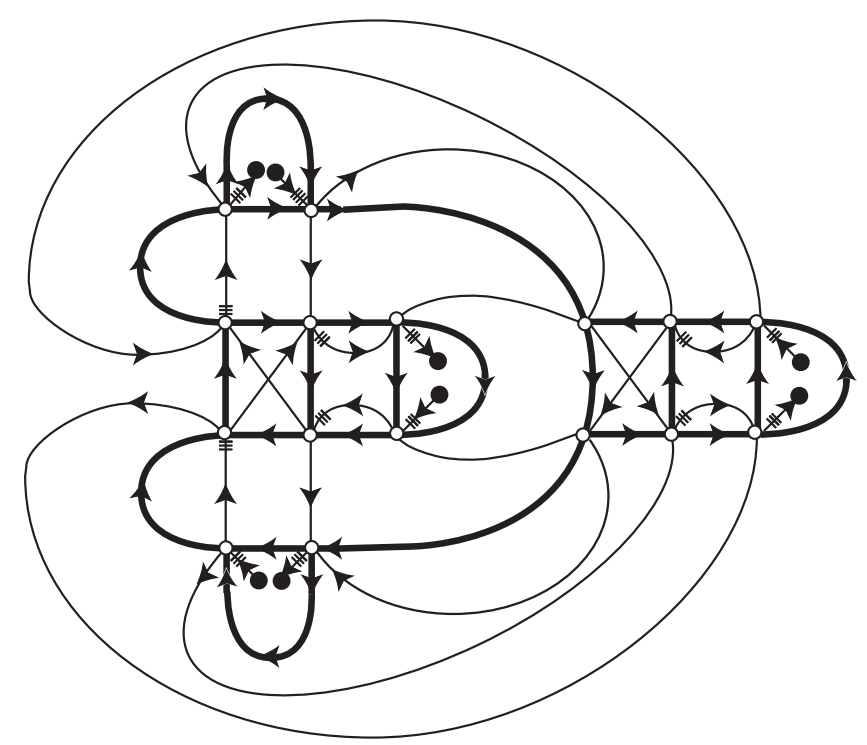

Fig. 14

\section{References}

[1] H. Aiba and T. Nagase, in preparation.

[2] J. S. Carter and M. Saito, Knotted Surfaces and their Diagrams, Math. Surveys Monogr. 55, Amer. Math. Soc., Providence, RI, 1998.

[3] S. Kamada, Surfaces in $R^{4}$ of braid index three are ribbon, J. Knot Theory Ramif. 1 (1992), 137-160.

[4] T. Nagase and A. Hirota, The closure of a surface braid represented by a 4-chart with at most one crossing is a ribbon surface, preprint.

Department of Mathematics

Tokai University

1117 Kitakaname, Hiratuka

Kanagawa, 259-1292 Japan

E-mail: nagase@keyaki.u-tokai.ac.jp

shima@keyaki.cc.u-tokai.ac.jp

Received 11 April 2004;

in revised form 22 September 2005 